\title{
Cost-effectiveness of intravitreal conbercept versus other treatments for wet age-related macular degeneration
}

\author{
Rui Chen ${ }^{1}$, Bin $W u^{2}$ \\ ${ }^{1}$ Department of Ophthalmology, The Second People's Hospital of Foshan, Foshan, China; ${ }^{2}$ Medical Decision and Economic Group, Department of \\ Pharmacy, Renji Hospital, School of Medicine, Shanghai Jiaotong University, Shanghai, China \\ Contributions: (I) Conception and design: B Wu; (II) Administrative support: B Wu; (III) Provision of study materials or patients: R Chen; (IV) \\ Collection and assembly of data: R Chen; (V) Data analysis and interpretation: All authors; (VI) Manuscript writing: B Wu; (VII) Final approval of \\ manuscript: All authors. \\ Correspondence to: Bin Wu. Department of Pharmacy, Renji Hospital, School of Medicine, Shanghai Jiao Tong University, Shanghai 200240, China. \\ Email: scilwsjtu-wb@yahoo.com.
}

Background: The potential benefits of conbercept, aflibercept, and ranibizumab has been reported in
patients with wet age-related macular degeneration (wAMD). However, their economic outcomes are still
unclear. The current study would assess the cost-effectiveness of conbercept, aflibercept and ranibizumab for
patients with wAMD in a Chinese healthcare setting. Methods: A Markov model was constructed based on patient visual acuity. Five regimens were considered: usual care without active anti-vascular endothelial growth factor (VEGF) treatment, IVT-AFL (intravitreal aflibercept on a two-monthly basis following three initial monthly doses), RBZ q4 (ranibizumab monthly dosing), RBZ RPN (ranibizumab dose as needed) and IVT-CON (intravitreal conbercept on a threemonthly basis after three initial monthly doses). Clinical, cost, and utility data were collected from published literature.

Results: In comparison with usual care, the IVT-AFL, RBZ q4, RBZ PRN, and IVT-CON strategies provided an additional $0.235,0.338,0.228$, and 0.324 quality-adjusted life years (QALYs), respectively. They had marginal costs of $\$ 6,800, \$ 10,084, \$ 4,640$, and $\$ 6,173$, respectively. The strategies also produced incremental cost-effectiveness ratios (ICERs) of \$28,892, \$29,857, \$20,338 and \$19,028/QALY, respectively. One-way sensitivity analysis showed utility of blindness (best-corrected visual acuity <35) to have the greatest sensitivity of all the parameters. Probabilistic sensitivity analysis (PSA) indicated that IVT-CON yielded the greatest probabilities of cost-effectiveness (about 92\%) compared with other strategies.

Conclusions: Conbercept is a cost-effective option for the treatment of wAMD in a Chinese healthcare setting.

Keywords: Age-related macular degeneration (AMD); ranibizumab; conbercept; aflibercept; cost-effectiveness

Submitted Feb 04, 2020. Accepted for publication Jun 23, 2020.

doi: $10.21037 /$ atm-20-1334

View this article at: http://dx.doi.org/10.21037/atm-20-1334

\section{Introduction}

Age-related macular degeneration (AMD), a chronic macular disease affecting the central retina, results in gradual loss and impairment of vision. According to the Global Burden of Disease Study 2017 (1), AMD was responsible for about 223,000 and 308,000 disabilityadjusted life years in men and women, respectively. Hand in hand with population aging, between 2007 to 2017, the disease burden was estimated to have risen by $34 \%$ and $28 \%$ in men and women, respectively. AMD can be classified as neovascular (wet or exudative) or nonneovascular (atrophic, dry, or non-exudative). Wet AMD (wAMD can affect central vision and with rapid progression over a period of weeks or months. Globally, it is one of the most prominent 


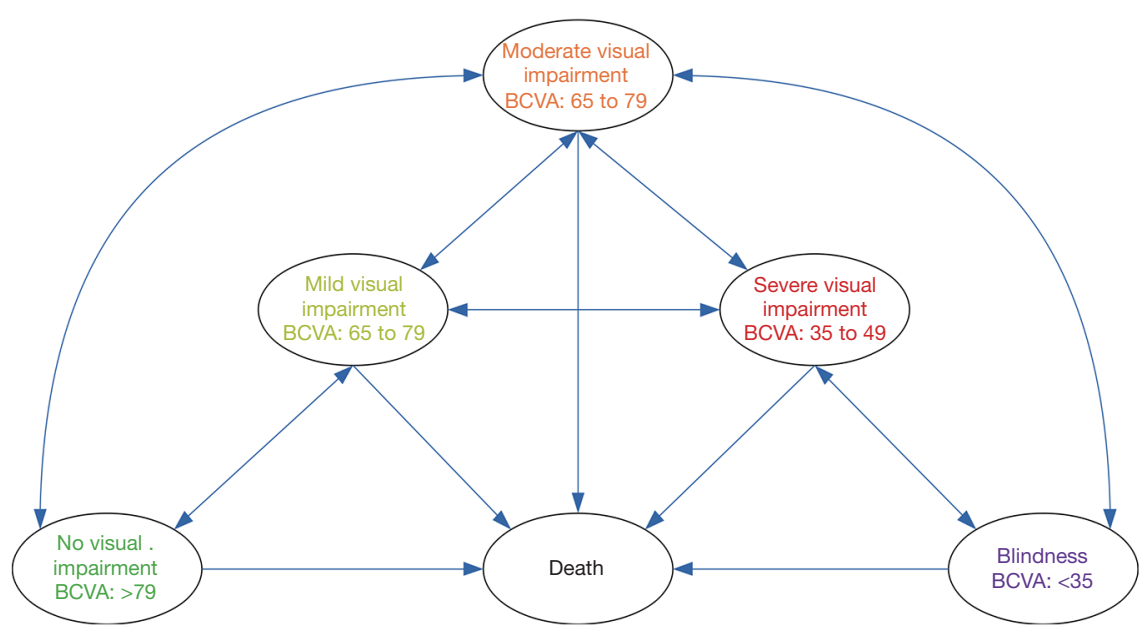

Figure 1 Overview of the Markov model structure. Health states are defined by BCVA. Patients have the risk of death in any state in the model. BCVA, best-corrected visual acuity.

causes of irreversible vision loss (2). AMD-associated blindness is currently one of the most challenging health issues across East Asia (3).

Vascular endothelial growth factor (VEGF), with its angiogenesis-regulating ability, carries out a key role in the pathogenesis of wAMD. Over the last decade, the treatment patterns of wAMD have been substantially reformed with the introduction of new anti-VEGF agents, such as aflibercept and ranibizumab, which have been proved to be superior to conventional treatments (4). In December 2013, conbercept, a recombinant fusion protein similar to aflibercept, with high affinity to all VEGF isoforms and PIGF, was developed and approved for the treatment of wAMD in China. One recent systematic review demonstrated conbercept to have comparable efficacy and safety profiles to ranibizumab (5). Moreover, the latest PHOENIX trial identified that a conbercept regimen of three initial monthly doses followed by quarterly treatments was effective for treating wAMD (6).

The potential benefits of conbercept, aflibercept, and ranibizumab reported in clinical trials were met with enthusiasm by ophthalmologists and patients alike. At present, both conbercept and ranibizumab have been frequently used in Chinese hospitals because they have become available for more than five year. Aflibercept also has been approved by Chinese National Medical Products Administration in 2018. However, their high cost is restrictive, and their widespread use would raise the socioeconomic burden on people and societies, particularly in areas where health resources are limited (7). Therefore, there is an urgent need for an economic evaluation of antiVEGF treatments in a clinical setting. In light of this, we carried out this evaluation of the cost-effectiveness of active anti-VEGF regimens for treating wAMD, in the context of a Chinese healthcare setting.

\section{Methods}

\section{Analytic overview}

We simulated the lifetime course of wAMD in hypothetical patients using a Markov model. The model structure, which is shown in Figure 1, included death state and five health states based on best-corrected visual acuity (BCVA) as follows: BCVA $>79$ (No visual impairment), 65 to 79 (Mild visual impairment), 65 to 79 (Moderate visual impairment), 65 to 79 (Severe visual impairment), and $<35$ (Blindness). During each one-month cycle of the Markov process, patients could remain in their state, or move to other health states. In each visual acuity state, patients were assumed to be in the median visual acuity for that state. Thus, an improvement by an average of 15 or 30 letters or a decrease by an average of 15 or 30 letters corresponded to a transition to the next higher or lower Markov state.

The characteristics of the hypothetical patients were assumed to be similar to those in the PHOENIX trial (6). All of the patients were newly diagnosed with wAMD and had a baseline age of 66 years. As China-specific data was not available, the initial BCVA distribution was gathered 
from published literature (8). Based on previous studies, the five following treatment strategies were assessed:

(I) Usual care without active therapy (reference strategy) $(9,10)$.

(II) Intravitreal aflibercept: $2 \mathrm{mg}$ loading dose monthly for the first 3 months followed by every other month until month 12, then capped PRN (retreatment upon predefined retreatment criteria, with a mandatory injection every 3 months) until month 24 (IVT-AFL) $(9,10)$.

(III) Intravitreal ranibizumab: $0.5 \mathrm{mg}$ dose monthly for 12 months, then capped PRN until month 24 (RBZ q4) $(9,10)$.

(IV) Intravitreal ranibizumab: $0.5 \mathrm{mg}$ loading dose monthly for the first 3 months followed by PRN until month 24 (RBZ PRN) $(9,10)$.

(V) Intravitreal conbercept: $0.5 \mathrm{mg}$ loading dose monthly for the first 3 months, then a dose once quarterly until month 24 (IVT-CON) (6).

In line with previous studies (9-11), the active treatments in the model were assumed up to 2 years.

Costs, quality-adjusted life years (QALYs), and length of blindness (years) were measured. A yearly discount rate of $5 \%$ was applied to both costs and QALYs. From these, we measured the incremental cost-effectiveness ratio (ICER; US \$ per additional QALY gained) of active anti-VEGF strategies in comparison with the usual care strategy. The maximum threshold limit for cost-effectiveness was set as $3 \mathrm{x}$ the Chinese gross domestic product (GDP) per capita in 2018 (\$28,410), based on the Chinese pharmacoeconomic guidelines (12).

\section{Clinical inputs}

The clinical profiles of each strategy were defined by the monthly proportions of gaining 15 to 30 letters and $>30$ letters, and losing 15 to 30 letters and $>30$ letters of vision. The annual probabilities of visual acuity change were transformed into monthly transition probabilities (Table 1). The clinical data of the IVT-AFL and RBZ q4 strategies were gathered from the Phase III VIEW 2 trial; estimates for the IVT-CON strategy were obtained from the PHOENIX trial (6); and the RBZ PRN strategy was extracted from the previous economic study (9). After discontinuation of active treatment, the probabilities of visual acuity loss were gathered from a meta-analysis of the natural history of wAMD and the sham arm of the MARINA trial $(13,14)$. Patients in the health states other than death would incur natural mortality, the data for which was obtained from the life tables of the World Health Organization (WHO) member states (2011). An excess risk of mortality was not assigned to patients with wAMD (9).

Low vision increases the risk of falls and depression $(15,16)$. The probabilities of depression and falls in each of the visual acuity categories were calculated by multiplying the risk ratios of each visual impairment category versus normal vision. The risk ratios and annual estimates of depression and falls were obtained based on our previous evaluation (11).

\section{Cost inputs}

This analysis was performed against the context of the Chinese healthcare system. Costs are presented in 2018 US dollar $(\$ 1=¥ 6.8)$. Only direct medical costs were considered, including the costs associated with wAMD management and the follow-up and direct medical costs associated with wAMD comorbidities (Table 2). The mean annual number of injections for conbercept, aflibercept, and ranibizumab was estimated based on published trials by calculating the weighted average $(6,8)$. The costs of drugs were obtained from a public databases (17), which presented the latest drug price in China. Due to the lack of dosage data in the second year, the number of injections for conbercept was set at 4 in the second year, corresponding with a trimonthly regimen recommendation. Beyond 2 years, the costs of anti-VEGF arms were assumed to be similar to those of the usual care strategy. For patients not receiving active anti-VEGF treatment, supportive care would be adopted. The cost was derived from the disease burden study, which involved 417 Chinese wAMD patients (18). The annual costs of comorbidities associated with low vision, including the depression and falls, were estimated based on our previous economic study (11).

\section{Health preferences}

Utility estimates were collected from a published study by Czoski-Murray et al. using a time trade-off methodology (Table 2) (19). Only the treatment effect in the better-seeing eye was considered in this study; compared to the poorerseeing eye, it shows more notable correlation between utility values and vision (20).

\section{Sensitivity analyses}

Sensitivity analyses are typically conducted to check that a 
Table 1 Clinical estimates

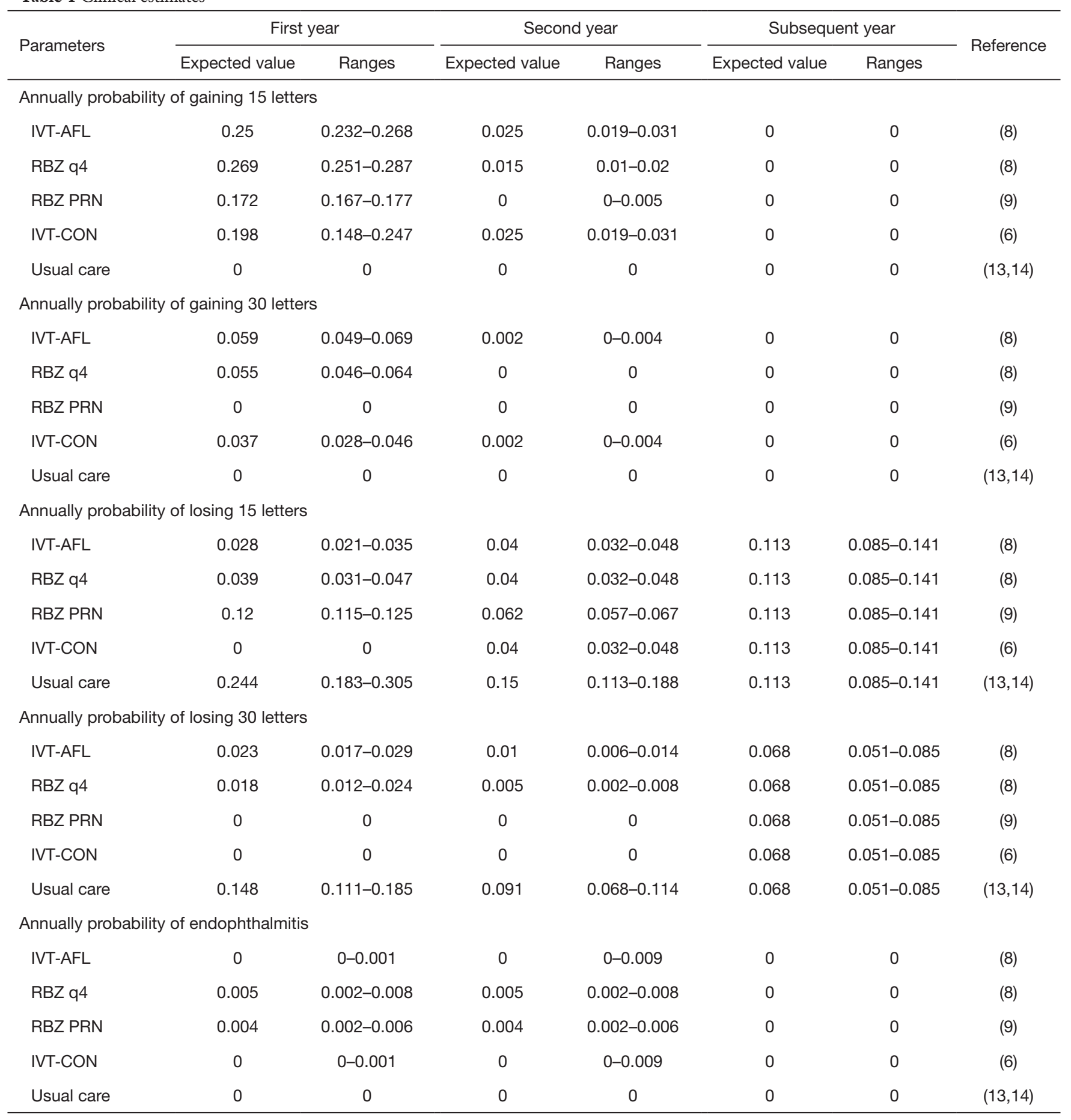

IVT-AFL, intravitreal aflibercept; RBZ q4, ranibizumab monthly dosing; RBZ RPN, ranibizumab dose as needed; IVT-CON, intravitreal conbercept. 
Table 2 Cost and utility estimates

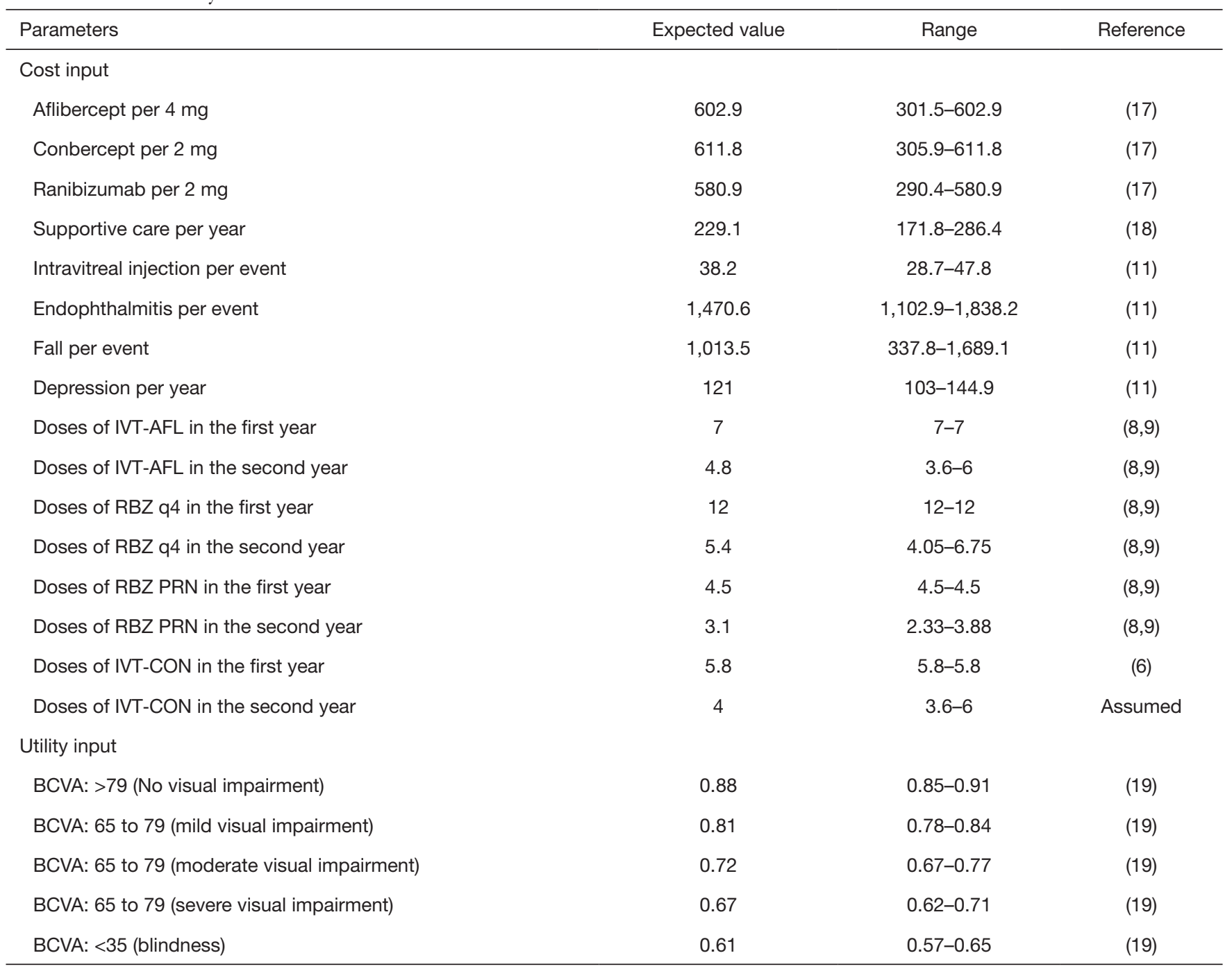

IVT-AFL, intravitreal aflibercept; RBZ q4, ranibizumab monthly dosing; RBZ RPN, ranibizumab dose as needed; IVT-CON, intravitreal conbercept; BCVA, best-corrected visual acuity.

model does not have any structural uncertainty and to assess the impact of outputs when specific variables are altered. By using the ranges shown in Tables 1,2 or assuming $\pm 25 \%$ of expected values, deterministic sensitivity analyses were carried out to test the impact of the parameters on the robustness of the results. A probabilistic sensitivity analysis (PSA) was performed to test the impact of uncertainty across all the parameters simultaneously by using a cohort of 1,000 second-order Monte Carlo simulations. Beta distribution was assigned for proportions, probabilities, hazard and risk ratios, and utilities, while lognormal distribution was used for costs. Based on the results of PSA, acceptability curves were plotted, which measured the willingness to pay (WTP) threshold for an incremental unit of effectiveness.

\section{Results}

\section{Base case analysis}

Compared with the usual care for wAMD patients (Table 3), the IVT-AFL, RBZ q4, RBZ PRN, and IVT-CON strategies saved 1.446, 2.406, 1.894 and 2.471 years of blindness with a lifetime horizon (Table 3), respectively. This translated to an additional $0.235,0.338,0.228$, and 0.324 QALYs respectively. The incremental costs of IVT-AFL, RBZ q4, RBZ PRN and IVT-CON against those of usual care were 
Table 3 Summary of cost $(\$)$ and outcome results in the base-case analysis

\begin{tabular}{lllcr}
\hline Strategies & Cost $(\$)$ & QALY & Years in blindness & ICER $(\$ / Q A L Y)^{*}$ \\
\hline Usual care & 31,154 & 6.552 & 7.846 & NA \\
IVT-AFL & 37,954 & 6.788 & 6.400 & 28,892 \\
RBZ q4 & 41,239 & 6.890 & 5.440 & 29,857 \\
RBZ PRN & 35,794 & 6.781 & 5.952 & 20,338 \\
IVT-CON & 37,327 & 6.877 & 5.375 & 19,028 \\
\hline
\end{tabular}

*, compared with usual care. QALY, quality-adjusted life year; ICER, incremental cost-effectiveness ratio; IVT-AFL, intravitreal aflibercept; RBZ q4, ranibizumab monthly dosing; RBZ RPN, ranibizumab dose as needed; IVT-CON, intravitreal conbercept.

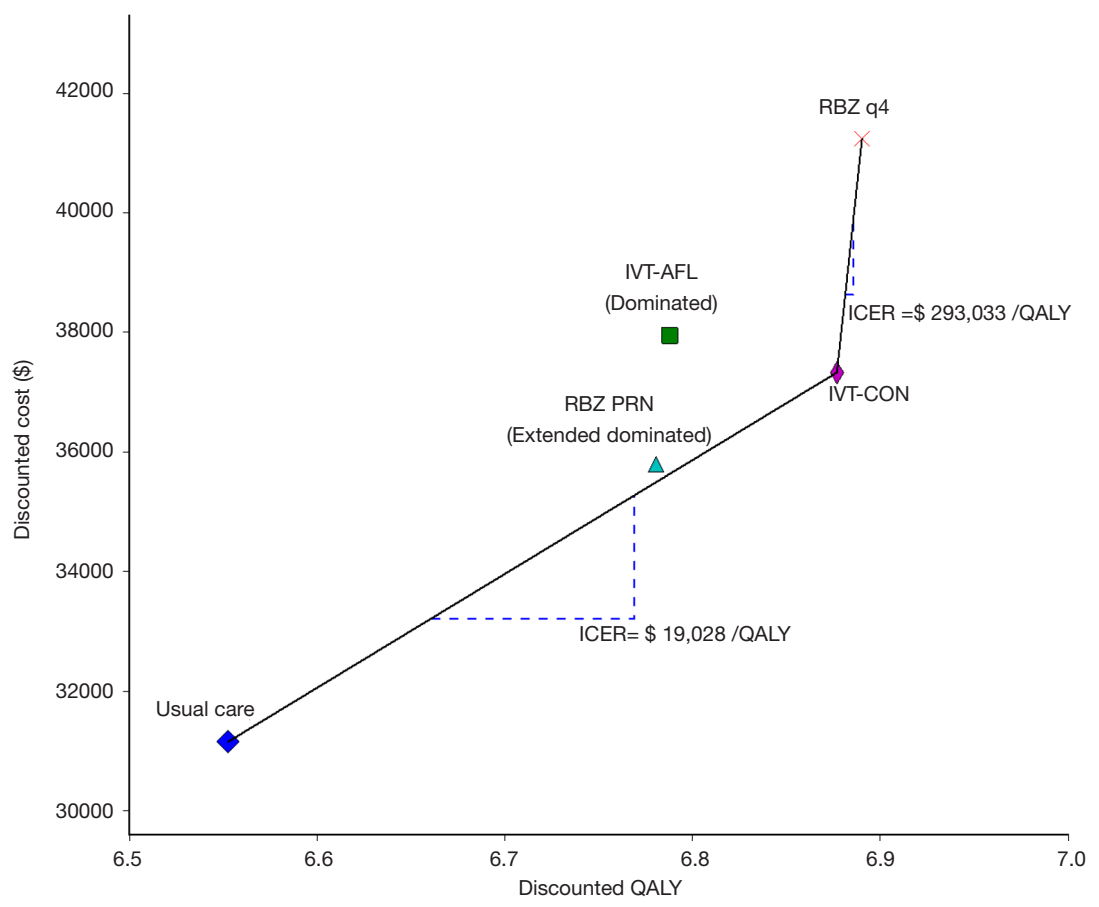

Figure 2 Cost-effective frontiers of different competing strategies. QALY, quality-adjusted life year; RBZ q4, ranibizumab monthly dosing; IV'T-AFL, intravitreal aflibercept; IVT-CON, intravitreal conbercept; RBZ RPN, ranibizumab dose as needed; ICER, incremental costeffectiveness ratio.

$\$ 6,800, \$ 10,084, \$ 4,640$ and $\$ 6,173$, respectively, which produced ICERs of $\$ 28,892$, $\$ 29,857$, $\$ 20,338$ and $\$ 19,028$ / QALY, respectively. The cost-effective frontier included the usual care, IVT-CON, and RBZ q4 strategies, and dominated IVT-AFL and extended dominated RBZ PRN strategies (Figure 2).

\section{Sensitivity analysis}

The deterministic sensitivity analyses of the IVT-CON strategy versus the usual care strategy showed that some model variables had a substantial impact on the results, which are presented in Figure 3. The variables with substantial influence included the utility of BCVA $<35$, the cost of conbercept, discount rate, RR of falls between blindness and normal vision, the utility of $50<$ BCVA $<64$, doses of conbercept in the second year, and patient age, which drove the ICERs over the threshold $(\$ 28,410 /$ QALY). Other variables, such as costs related to falls and depression, had moderate or little impact on the model 


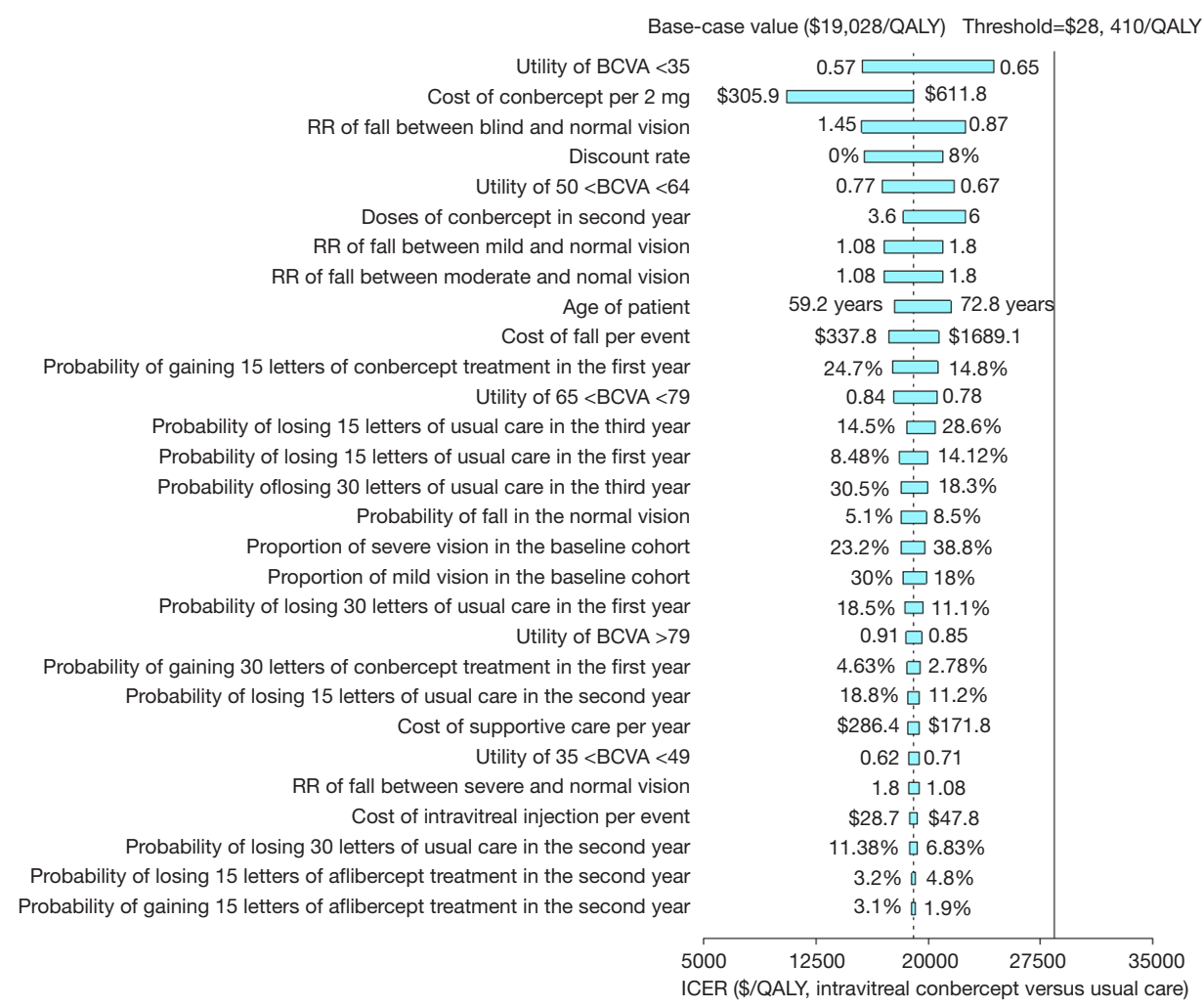

Figure 3 Tornado diagrams showing the affection of lower and upper values of each parameter in the incremental cost-effectiveness ratio of the IVT-CON versus usual care strategy. QALY, quality-adjusted life year; BCVA, best-corrected visual acuity; IVT-CON, intravitreal conbercept.

outputs.

Based on PSA, acceptability curves showed that wAMD treatment with IVT-CON yielded acceptable ICERs at the \$28,410/QALY threshold in most (about 92\%) cases compared with the usual care, IVT-AFL, RBZ q4, and RBZ PRN strategies (Figure 4).

\section{Discussion}

To our knowledge, this evaluation is the first to conduct an economic analysis of the costs and health outcomes of conbercept, aflibercept, and ranibizumab and to compare them with those of usual care. The results of our analysis indicated that the cost per QALY gained by intravitreal conbercept treatment against usual care with a lifetime horizon is $\$ 25,849$, just below $3 \times$ GDP per capita of China (\$28,410 in 2018), making it a cost-effective option. However, other anti-VEGF drugs, including aflibercept and ranibizumab, did not present as cost-effective alternatives due to their unfavorable ICERs compared to that of usual care. These findings were further strengthened by PSA.

The results of one-way sensitivity analysis suggested that the utility of BCVA $<35$ was the main driver of the economic outcomes, which indicates that anti-VEGF treatment would generate more cost-effective outcomes in patients with lower health preference of BCVA $<35$. One recent quality-of-life study revealed age to be a significant negative parameter of utility value (21). Because of the high prevalence of AMD among older people (>75 years) (22), it could be suggested that anti-VEGF treatment would prove to be more cost-effective in the older population.

One ISPOR 2015 meeting abstract showed that conbercept RPN is a superior cost-saving alternative for treating wAMD in Chinese people over a one-year time horizon, compared with monthly ranibizumab (23); this is inconsistent with our findings and could potentially be attributed to the price of anti-VEGF agents and clinical inputs. The price of conbercept $(\$ 988 / 0.5 \mathrm{mg})$ was about $30 \%$ lower than ranibizumab $(\$ 1,426 / 0.5 \mathrm{mg})$ in 2015 . However, after the recent pricing negotiations with the 


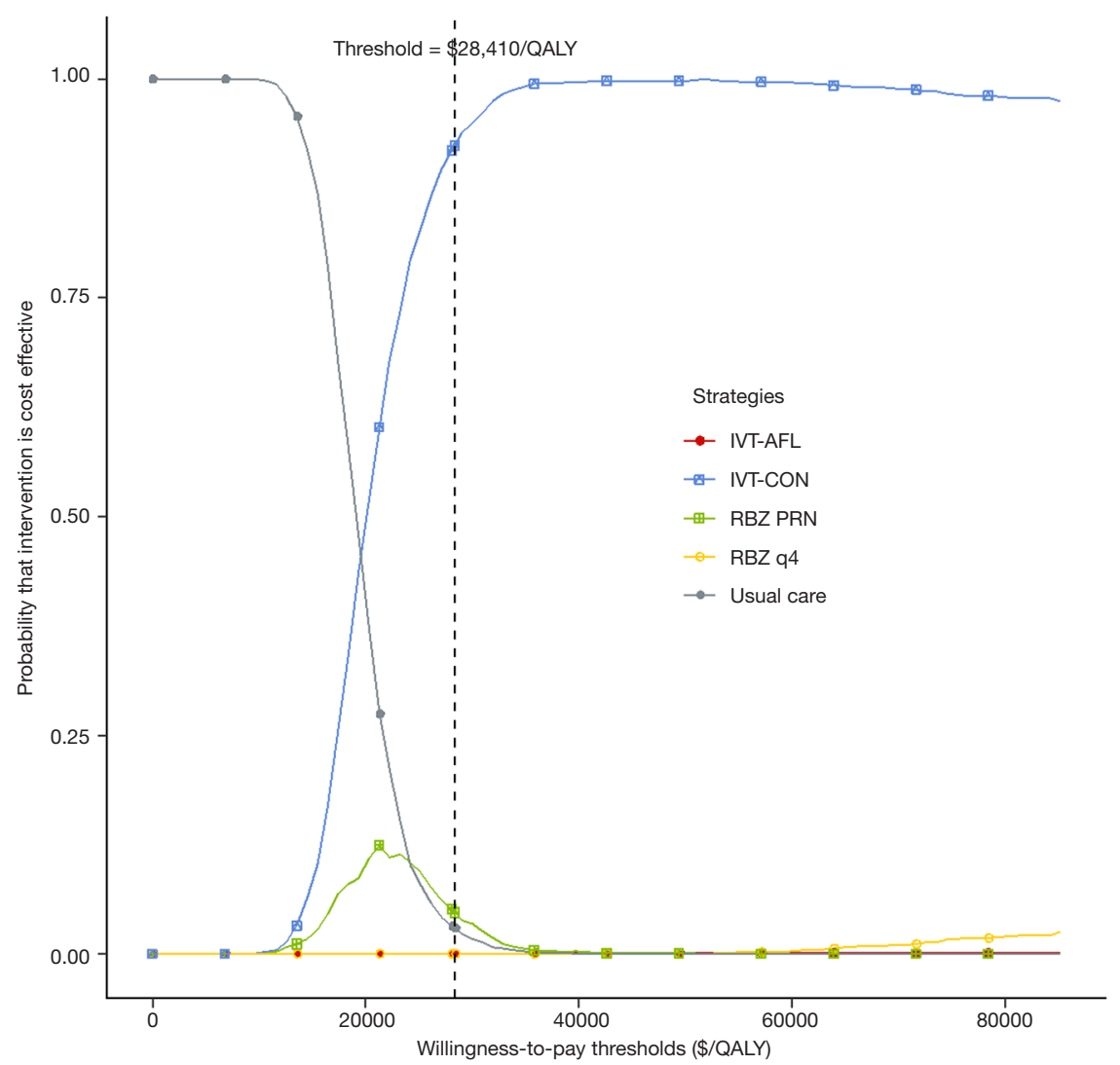

Figure 4 Acceptability curves comparing the cost-effectiveness of different competing strategies. The threshold is US $\$ 28,410(3 \times$ the gross domestic product per capita of China in 2018). QALY, quality-adjusted life year; IVT-AFL, intravitreal aflibercept; IVT-CON, intravitreal conbercept; RBZ RPN, ranibizumab dose as needed; RBZ q4, ranibizumab monthly dosing.

Chinese healthcare insurance system in 2019 (24), the prices of aflibercept, ranibizumab, and conbercept were substantially reduced, leading to a small difference between the prices of ranibizumab and conbercept. The efficacy data of the meeting abstract were extracted from the EXTEND II and AURORA trials, which were single-arm and controlled-dose studies, respectively, designed with only one-year follow-up time. Due to the heterogeneity of our study design and the patients' baseline characteristics, the clinical efficacy data of the EXTEND II and AURORA trials could not be directly input into our model. Consequentially, these mode inputs and design led to our analysis producing cost-effective results, rather than the cost-saving results referred to in the meeting abstract.

Several economic evaluations have been performed showing aflibercept to be cheaper than RBZ q4, while demonstrating similar efficacy. Aflibercept has proved to be more cost-effective than RBZ PRN in the United States,
Japan, Sweden, and the Netherlands $(9,10,25,26)$. Although our analysis also determined aflibercept to be cheaper than RBZ q4 in a Chinese healthcare setting, the ICER of aflibercept versus RBZ PRN was \$446,942/QALY, which indicates that the aflibercept strategy is not cost-effective in comparison with the RBZ PRN strategy in China. Other economic evaluations have also found that bevacizumab is more cost-effective than both ranibizumab and aflibercept $(27,28)$. However, our analysis only included anti-VEGF agents with indications, and bevacizumab has not yet been approved in China for treating AMD. Our previous evaluation found that, in a Chinese healthcare context, bevacizumab might serve as a better a cost-saving option than ranibizumab (11). With limited health care budgets, it is important to present realistic estimates of cost. Therefore, it should be noted that these reports were performed in high-income regions, which generally have better-funded healthcare resources and a higher threshold than middle 
and low-income regions, such as China. Our findings might provide some economic evidence to inform the decisions of patients, physicians, and policymakers from middle and low-income regions.

This analysis has several weaknesses that need to be considered along with its results. Firstly, because of a lack of direct head-to-head studies comparing aflibercept, ranibizumab, and conbercept with usual care, this study adopted an indirect approach in assessing the economic outcomes of the five strategies. In turn, this could have yielded inevitable bias in our findings due to the resulting study design and patient characteristics. Future evaluations should be updated if direct comparison data becomes available. Secondly, because of the lack of Chinese-specific inputs, especially the clinical profiles of aflibercept and ranibizumab and heath preference data, the present study mainly used data extracted from literature reported abroad, which might have contributed a degree of uncertainty. Recent subgroup analyses from the VIEW trials showed that the outcomes of intravitreal aflibercept, and ranibizumab among Asian patients are comparable to those among Caucasian patients (29). However, Chinese AMD patients and those in Western populations generally experience different quality of life $(30,31)$. Thirdly, the current study did not test the 'continuous treatment effect' approach, under which anti-VEGF treatment would be administered beyond two years, because the efficacy of this approach is still unknown. Fourthly, this analysis did not include indirect costs, such as the loss of productivity, which could lead to a considerable social economic burden for a patient's family and society. If these indirect costs were included, the cost-effectiveness of active anti-VEGF treatment would become more favorable. Fifthly, the current analysis did not consider other potential treatments, such as bevacizumab, which is not approve in Chinese clinical practice. Finally, to keep the model streamlined, reduced utility due to adverse events was not factored in. However, because adverse events are both uncommon and mild with conbercept, aflibercept, and ranibizumab, this had little impact on the outcomes.

\section{Conclusions}

In conclusion, although conbercept, aflibercept, and ranibizumab are licensed for the treatment of wAMD in China, conbercept is the only option more cost-effective than usual care due to its favorable economic outcomes in the current Chinese healthcare setting. Therefore, conbercept should be preferred for treating the Chinese patients with wAMD by weighting the costs and clinical benefits. To alleviate the financial burden inflicted by wAMD, the price of anti-VEGF drugs should be reduced.

\section{Acknowledgments}

Funding: None.

\section{Footnote}

Conflicts of Interest: Both authors have completed the ICMJE uniform disclosure form (available at http://dx.doi. org/10.21037/atm-20-1334). The authors have no conflicts of interest to declare.

Ethical Statement: The authors are accountable for all aspects of the work in ensuring that questions related to the accuracy or integrity of any part of the work are appropriately investigated and resolved.

Open Access Statement: This is an Open Access article distributed in accordance with the Creative Commons Attribution-NonCommercial-NoDerivs 4.0 International License (CC BY-NC-ND 4.0), which permits the noncommercial replication and distribution of the article with the strict proviso that no changes or edits are made and the original work is properly cited (including links to both the formal publication through the relevant DOI and the license). See: https://creativecommons.org/licenses/by-nc-nd/4.0/.

\section{References}

1. GBD 2017 DALYs and HALE Collaborators. Global, regional, and national disability-adjusted life-years (DALYs) for 359 diseases and injuries and healthy life expectancy (HALE) for 195 countries and territories, 1990-2017: a systematic analysis for the Global Burden of Disease Study 2017. Lancet 2018;392:1859-922.

2. Mitchell P, Liew G, Gopinath B, et al. Age-related macular degeneration. Lancet 2018;392:1147-59.

3. Cheng CY, Wang N, Wong TY, et al. Prevalence and causes of vision loss in East Asia in 2015:magnitude, temporal trends and projections. Br J Ophthalmol 2020;104:616-22.

4. Alexander M, Halmos B. VEGF inhibitors in EGFRmutated lung cancer: a never-ending story? Ann Transl Med 2018;6:446.

5. Zhang J, Liang Y, Xie J, et al. Conbercept for patients 
with age-related macular degeneration:a systematic review. BMC Ophthalmol 2018;18:142.

6. Liu K, Song Y, Xu G, et al. Conbercept for Treatment of Neovascular Age-related Macular Degeneration:Results of the Randomized Phase 3 PHOENIX Study. Am J Ophthalmol 2019;197:156-67.

7. Schmidt-Erfurth U, Chong V, Loewenstein A, et al. Guidelines for the management of neovascular agerelated macular degeneration by the European Society of Retina Specialists (EURETINA). Br J Ophthalmol 2014;98:1144-67.

8. Schmidt-Erfurth U, Kaiser PK, Korobelnik JF, et al. Intravitreal aflibercept injection for neovascular agerelated macular degeneration: ninety-six-week results of the VIEW studies. Ophthalmology 2014;121:193-201.

9. Panchmatia HR, Clements KM, Hulbert E, et al. Aflibercept vs. Ranibizumab: cost-effectiveness of treatment for wet age-related macular degeneration in Sweden. Acta Ophthalmol 2016;94:441-8.

10. Hernandez L, Lanitis T, Cele C, et al. Intravitreal Aflibercept Versus Ranibizumab for Wet Age-Related Macular Degeneration: A Cost-Effectiveness Analysis. J Manag Care Spec Pharm 2018;24:608-16.

11. Wu B, Li J, Lin H, et al. Different Strategies for the Treatment of Age-Related Macular Degeneration in China: An Economic Evaluation. J Ophthalmol 2016;2016:7689862.

12. Xiao J, Sun JF, Wang QQ, et al. Health economic evaluation reporting guideline and application status. Zhonghua Yu Fang Yi Xue Za Zhi 2017;51:276-80.

13. Shah AR, Del PL. Progressive visual loss in subfoveal exudation in age-related macular degeneration: a metaanalysis using Lineweaver-Burke plots. Am J Ophthalmol 2007;143:83-9.

14. Rosenfeld PJ, Brown DM, Heier JS, et al. Ranibizumab for neovascular age-related macular degeneration. N Engl J Med 2006;355:1419-31.

15. Earnshaw SR, Moride Y, Rochon S. Cost-effectiveness of pegaptanib compared to photodynamic therapy with verteporfin and to standard care in the treatment of subfoveal wet age-related macular degeneration in Canada. Clin Ther 2007;29:2096-106; discussion 2094-5.

16. Javitt JC, Zhou Z, Willke RJ. Association between vision loss and higher medical care costs in Medicare beneficiaries costs are greater for those with progressive vision loss. Ophthalmology 2007;114:238-45.

17. Anonymous. The Database of Drug Price. Available online: https://www.yaozh.com/. Accessed July 26, 2019.
18. Zhang YB, Hu SL, Chang JH. Analysis on the Disease Burden of Wet Age-related Macular Degeneration in China. Chinese Health Economics 2015;34:63-5.

19. Czoski-Murray C, Carlton J, Brazier J, et al. Valuing condition-specific health states using simulation contact lenses. Value Health 2009;12:793-9.

20. Hodgson R, Reason T, Trueman D, et al. Challenges Associated with Estimating Utility in Wet Age-Related Macular Degeneration: A Novel Regression Analysis to Capture the Bilateral Nature of the Disease. Adv Ther 2017;34:2360-70.

21. Visser MS, Amarakoon S, Missotten T, et al. SF-6D utility values for the better- and worse-seeing eye for health states based on the Snellen equivalent in patients with agerelated macular degeneration. Plos One 2017;12:e0169816.

22. Jonas JB, Cheung C, Panda-Jonas S. Updates on the Epidemiology of Age-Related Macular Degeneration. Asia Pac J Ophthalmol (Phila) 2017;6:493-7.

23. Zhao M, Feng W, Zhang L, et al. PSS34 - CostEffectiveness Analysis of Conbercept Versus Ranibizumab For The Treatment of Age-Related Macular Degeneration In China. Value Health 2015;18:A421.

24. Li H, Liu GG, Wu J, et al. Recent Pricing Negotiations on Innovative Medicines Pilot in China: Experiences, Implications, and Suggestions. Value Health Reg Issues 2018;15:133-7.

25. Yanagi Y, Fukuda A, Barzey V, et al. Cost-effectiveness of intravitreal aflibercept versus other treatments for wet age-related macular degeneration in Japan. J Med Econ 2017;20:204-12.

26. Elshout M, van der Reis MI, Webers CA, et al. The costutility of aflibercept for the treatment of age-related macular degeneration compared to bevacizumab and ranibizumab and the influence of model parameters. Graefes Arch Clin Exp Ophthalmol 2014;252:1911-20.

27. van Asten F, Michels C, Hoyng CB, et al. The costeffectiveness of bevacizumab, ranibizumab and aflibercept for the treatment of age-related macular degeneration-A cost-effectiveness analysis from a societal perspective. Plos One 2018;13:e0197670.

28. Elshout M, Webers C, van der Reis MI, et al. A systematic review on the quality, validity and usefulness of current cost-effectiveness studies for treatments of neovascular age-related macular degeneration. Acta Ophthalmol 2018;96:770-8.

29. Wong TY, Cheung C, Lai T, et al. Efficacy and safety of intravitreal aflibercept and ranibizumab in Asian patients with neovascular age-related macular degeneration: 
Subgroup Analyses From the VIEW Trials. Retina 2019;39:537-47.

30. Bian W, Wan J, Smith G, et al. Domains of health-related quality of life in age-related macular degeneration: a qualitative study in the Chinese cultural context. BMJ

Cite this article as: Chen $\mathrm{R}, \mathrm{Wu}$ B. Cost-effectiveness of intravitreal conbercept versus other treatments for neovascular age-related macular degeneration. Ann Transl Med 2020;8(15):939. doi: 10.21037/atm-20-1334
Open 2018;8:e018756.

31. Au Eong KG, Chan EW, Luo N, et al. Validity of EuroQOL-5D, time trade-off, and standard gamble for age-related macular degeneration in the Singapore population. Eye (Lond) 2012;26:379-88. 